(BASHH) standards for the management of STIs recommends treatment "in as short a timescale as possible". The National Chlamydia Screening Programme (NCSP) sets a key indicator of treating $\geq 95 \%$ of those testing positive within six weeks of test date.

Aim(s)/objectives To explore the feasibility of services achieving a shorter time to treatment standard.

Methods National audit data from the most recent NCSP turnaround time audit were used to explore how many services would meet treatment targets of three and two weeks from test date.

Results The current time to treatment standard of $\geq 95 \%$ treated within six weeks was achieved by $39 \%$ of providers $191 \%$ of positive patients receiving treatment within six weeks, due to large services having a proportionately greater impact). Using the targets of three and two weeks this fell to $28 \%$ and $4 \%$ of providers, respectively. However, this represents $88 \%$ of patients treated within three weeks and $76 \%$ within two weeks (Table 1).

\begin{tabular}{lll} 
Abstract P158 Table 1 & \multicolumn{2}{c}{ Chlamydia treatment } \\
\hline & $\begin{array}{l}\% \text { of patients } \\
\text { treated within the } \\
\text { timescale }\end{array}$ & $\begin{array}{l}\% \text { of providers } \\
\text { with } \geq 95 \% \text { of patients } \\
\text { treated within the timescale }\end{array}$ \\
Timescale & $91 \%$ & $39 \%$ \\
\hline Six working weeks & $88 \%$ & $28 \%$ \\
Three working weeks & $76 \%$ & $4 \%$ \\
Two working weeks &
\end{tabular}

Discussion/conclusion $88 \%$ of positive patients were treated within three weeks from test date even though only $28 \%$ of providers would have been able to meet this time to treatment standard. Meeting a shorter time to treatment standard would be challenging but could help to drive quality improvement and may form part of updated standards for the NCSP.

\section{P159 SEXUAL HEALTH SERVICES ARE IDEALLY PLACED TO MANAGE VULNERABLE YOUNG PEOPLE?}

Rhian Willson*, Justine Orme, Deborah Williams, Daniel Richardson. Brighton and Sussex University Hospital NHS Trust, Brighton, UK

\subsection{6/sextrans-2015-052126.202}

Background/introduction Identifying and assessing the risk of child sexual exploitation (CSE) in young people is a fundamental role of sexual health clinics. The 'Spotting the signs' proforma developed by BASHH recommends assessing all those $<18$ yrs for risk factors.

Aim(s)/objectives The aim of this audit was to review those $<18$ yr olds attending the GU clinic in Brighton assessed as medium or high risk to investigate the areas of concern, the appropriateness of interventions and follow up.

Methods EPR records for all $<18$ yr olds between 1/4/14 and 31/10/14 were reviewed.

Results 56 patients identified, 86 attendances. 36/56 (64\%) were 16-17 yrs. 48/56 (86\%) were female. 23/56 (41\%) were seen in the Young Person's Clinic, the rest seen throughout the service. Concerns included: sexual assault/non-consensual sex $41 \%$, drugs and alcohol 39\%, difficulties at home/in care 37\%, mental health $37 \%$ and partner age/coercion 11\%. 20\% had concerns in $\geq 3$ areas. Interventions: 24/56 (53\%) already had social work or other agency involvement, $27 \%$ were referred to agencies for the first time as a consequence of their visit to the clinic. Further clinic follow up was arranged in 33/56 (59\%). All patients had a clear action plan.
Discussion/conclusion This audit suggests that older young people (16-17 yrs) have significant risk factors; the same vigilance accorded to under 16's needs to be applied to this group. Sexual Health clinics are well placed to both recognise those at risk and provide ongoing support and referral.

\section{P160 WHAT IMPACT DID THE XX COMMONWEALTH GAMES HAVE ON STIS AND SEXUAL HEALTH SERVICES IN GLASGOW?}

Rebecca Acquah*, Rebecca Metcalfe, Andrew Winter. Sandyford Sexual Health Services, NHS Greater Glasgow and Clyde, Glasgow, UK

\subsection{6/sextrans-2015-052126.203}

Background/introduction An estimated 600,000 spectators, volunteers and athletes from over 70 countries visited Glasgow for the XX Commonwealth Games, held between 23 July and 3 August 2014, doubling the city's population.

Aim(s)/objectives We sought to investigate the impact of the Games on the number of acute STIs and on service activity in core specialist sexual health services, which offer free walk-in access.

Methods We interrogated our city-wide electronic patient record system $(\mathrm{NaSH})$ to measure service activity, the number of acute STIs and PEPSE prescriptions between the $9^{\text {th }}$ July and the $31^{\text {st }}$ August 2014. We compared these to the same time period in 2013. We prospectively asked all new clinic attendees if they were in Glasgow for the Games.

Results

\begin{tabular}{llll}
\hline & \multicolumn{2}{c}{ Games } & \\
\cline { 2 - 3 } Results & $\mathbf{2 0 1 4}$ & $\mathbf{2 0 1 3}$ & Difference \\
\hline Total Attendances & 14,973 & 16,440 & $-8.9(\downarrow)$ \\
New Registrations & 1,986 & 2,150 & $-7.6(\downarrow)$ \\
Acute STI episode & 623 & 693 & $-10.1(\downarrow)$ \\
Gonorrhoea & 78 & 81 & $-3.7(\downarrow)$ \\
Chlamydia & 372 & 428 & $-13.1(\downarrow)$ \\
Early syphilis & 15 & 14 & $7.1(\uparrow)$ \\
NSU & 83 & 78 & $6.4(\uparrow)$ \\
Trichomonas & 3 & 2 & $50.0(\uparrow)$ \\
Primary HSV & 64 & 79 & $-19.0(\downarrow)$ \\
PEPSE prescriptions & 8 & 11 & $-27.3(\downarrow)$ \\
\hline
\end{tabular}

Of the 1496 attendees who responded, just 1.7\% (26) were in Glasgow solely for the Games.

Discussion/conclusion Despite the huge influx of visitors, service activity and overall acute symptomatic STI incidence decreased by around 10\% during and after the Games compared to 2013. We found no evidence that large sporting events increase demand for sexual health services or cause a rise in acute STIs.

\section{P161 SEXUAL HEALTH IN GENERAL PRACTICE: DO GP PRACTICES COMPLY WITH BASHH GUIDELINES?}

Craig Trotter, Yetunde Okunwobi-Smith*. Cumbria Partnership NHS Foundation Trust, Cumbria, UK

\subsection{6/sextrans-2015-052126.204}

Background The passing of the Health and Social Care Act 2012 committed to more services in the community provided closer to home and by GPs. Therefore most GP practices are commissioned to provide Level one STI screening. 